OPEN ACCESS

Edited by:

Yanhui Liao

Sir Run Run Shaw Hospital, China

Reviewed by:

Kenji Hashimoto,

Chiba University, Japan

Ni Fan,

The Affiliated Brain Hospital of Guangzhou Medical University, China

${ }^{*}$ Correspondence: Hongxian Shen

shenhx2018@csu.edu.cn

Xiaojie Zhang

xiaojiezhang2014@163.com

${ }^{\dagger}$ These authors have contributed equally to this work and share first authorship

Specialty section:

This article was submitted to Addictive Disorders, a section of the journal

Frontiers in Psychiatry

Received: 25 June 2020 Accepted: 02 September 2020 Published: 25 September 2020

Citation: Li M, Xie A, Liu Y, Zeng Q, Huang S, Huang $Q$, Shao $T$, Chen $X$, Liao Z,

Cai $Y$, Xiao Z, Zhang $X$ and Shen $H$ (2020) Ketamine Administration Leads to Learning-Memory Dysfunction and

Decreases Serum Brain-Derived

Neurotrophic Factor in Rats.

Front. Psychiatry 11:576135. doi: 10.3389/fpsyt.2020.576135

\section{Ketamine Administration Leads to Learning-Memory Dysfunction and Decreases Serum Brain-Derived Neurotrophic Factor in Rats}

\author{
Miao $\mathrm{Li}^{1,2,3,4 \dagger}$, Aiming Xie ${ }^{5 \dagger}$, Ya Liu ${ }^{3,6}$, Qian Zeng ${ }^{3}$, Shucai Huang ${ }^{7}$, Qiuping Huang ${ }^{1,2}$, \\ Tianli Shao ${ }^{1,2}$, Xinxin Chen $^{1,2}$, Zhenjiang Liao ${ }^{1,2}$, Yi Cai $^{8}$, Zhijie Xiao $^{3}$, Xiaojie Zhang ${ }^{1,2 *}$ \\ and Hongxian Shen ${ }^{1,2 *}$

\begin{abstract}
${ }^{1}$ Department of Psychiatry, The Second Xiangya Hospital of Central South University, and National Clinical Research Center for Mental Disorders, Changsha, China, ${ }^{2}$ Institute of Mental Health of Central South University, Chinese National Technology Institute on Mental Disorders, Hunan Key Laboratory of Psychiatry and Mental Health, Hunan Medical Center for Mental Health, Changsha, China, ${ }^{3}$ Department of Neurology, The Second Xiangya Hospital, Central South University, Changsha, China, ${ }^{4}$ Department of Neurology, The First Hospital of Changsha, Changsha, China, ${ }^{5}$ Affiliated Wuhan Mental Health Center, Tongji Medical College of Huazhong University of Science \& Technology, Wuhan, China, ${ }^{6}$ Department of Neurology, Changsha Central Hospital, Changsha, China, ${ }^{7}$ Department of Psychiatry, The Fourth People's Hospital of Wuhu, Wuhu, China, ${ }^{8}$ Department of Psychiatry, Geriatric and Somatic Diseases, Brain Hospital of Human Province, Hunan University of Chinese Medicine Clinical College, Hunan Mental Behavior Disorder Research Center, Changsha, China
\end{abstract}

Objective: This study investigated the effects of acute or chronic ketamine administration on learning and memory function as well as levels of brain-derived neurotrophic factor (BDNF) in the hippocampus and blood in order to explore the potential correlation between learning-memory dysfunction and ketamine.

Methods: Rats were treated with $25 \mathrm{mg} / \mathrm{kg}$ ketamine for $3 \mathrm{~d}(\mathrm{n}=20)$ or $14 \mathrm{~d}(\mathrm{n}=20)$. Saline-treated rats were used as controls. The Morris water maze test was used to evaluate spatial learning and memory after $10 \mathrm{~d}$ of withdrawal. The level of BDNF in serum and the hippocampus were measured by ELISA.

Results: The number of platform crossings and residence time in the target platform quadrant were significantly reduced in ketamine $3 \mathrm{~d}$ and $14 \mathrm{~d}$ groups than in the saline controls (both $p<0.05$ ). In addition, the average escape latency of ketamine $3 d$ and $14 d$ groups were significantly longer than that of the saline $3 d$ and $14 d$ groups $(p<0.0001)$, respectively. Further examination found that only serum samples from ketamine $14 \mathrm{~d}$ group showed significantly decreased BDNF level compared to that from saline $14 \mathrm{~d}$ groups $(p<0.05)$. However, no differences were detected in hippocampus samples.

Conclusion: Chronic ketamine exposure $(25 \mathrm{mg} / \mathrm{kg}$ ) causes spatial learning and memory deficits in SD rats, which may be associated with decreased serum BDNF levels.

Keywords: ketamine administration, spatial learning, memory, Morris water maze, brain-derived neurotrophic factor 


\section{INTRODUCTION}

Ketamine is a commonly studied psychoactive substance and known drug of abuse. Recently, it has been widely abused as a new type of drug (also known as $\mathrm{K}$ powder) due to its addictive properties $(1,2)$. Ketamine abuse can lead to cognitive impairment $(3,4)$. Ketamine is an antagonist of the N-methyl-D-aspartate (NMDA) receptor, and can lead to similar symptoms of addiction (5). Recent studies have shown that low-dose ketamine does not have a significant effect on cognition function (6), but sub-anesthetic dose and anesthetic-dose ketamine could cause cognitive impairment by affecting executive function, procedural function, episodic memory, semantic memory, and working memory $(2,6,7)$. Importantly, the effects of ketamine vary greatly with duration. In humans, acute ketamine treatment is known to impair episodic and working memory, recognition memory, and procedural learning (7). This has been further confirmed in mice, as chronic ketamine treatment also leads to memory impairment (8). Ketamine can induce behavioral changes in users and this change is related to the frequency and dose of use (9).

Brain-derived neurotrophic factor (BDNF), abundant in the central nervous system, is known to regulate synaptic plasticity as well as participate in learning and memory processes. BDNF is also likely to participate in the pathophysiology of drug addiction, including the cognitive impairment (10-12). In a study by $\mathrm{Ke}$ et al. serum BDNF concentrations were significantly lower in chronic ketamine users than in health controls (2). Importantly, it has been found that the level of BDNF in blood can be used to approximate protein concentration changes in brain tissues (12). It is therefore likely that BDNF concentrations are similarly lower in the brain of chronic ketamine users, particularly within the reward system of the brain. This network of brain structures, namely the ventral tegmental area of the midbrain and its connections to the nucleus accumbens and prefrontal cortex, governs reward, and is well established to be involved in drug addiction (13). In addition to reward circuity, exposure to drugs of abuse, including ketamine, can alter plasticity in cognition as well as learning and memory-related brain areas. Importantly, these changes can persist long after drug exposure has ceased (14).

Therefore, in order to further elucidate the potential relationship between BDNF and ketamine-induced learningmemory dysfunction, we investigated the effects of acute and chronic ketamine $(25 \mathrm{mg} / \mathrm{kg})$ on cognition as well as BDNF levels in the blood and hippocampus of Sprague-Dawley (SD) rats.

\section{MATERIALS AND METHODS}

\section{Animals}

A total of 75 adult healthy male SD rats were randomly divided into four groups: ketamine $3 \mathrm{~d}$ group $(\mathrm{n}=20)$, saline 3 d group $(n=18)$, ketamine 14 d group $(n=20)$, saline 14 d group $(n=17)$. The experiment was carried out after a week of adaptive feeding.

\section{Ketamine Exposure}

All rats were intraperitoneally injected with ketamine, $25 \mathrm{mg} / \mathrm{kg}$ for either $3 \mathrm{~d}$ or $14 \mathrm{~d}$, and the saline group was injected with
$0.9 \%$ saline for $3 \mathrm{~d}$ or $14 \mathrm{~d}$ as controls. Injections were performed daily at 4:30 p.m. In order to avoid the effect of withdrawal reaction on behavioral experiments, the rats were given a 10-day withdrawal period.

\section{Morris Water Maze (MWM) Test}

MWM test was conducted in a round stainless steel water tank with a diameter of $1.2 \mathrm{~m}$ and a height of $0.5 \mathrm{~m}$, with an attached tracking and recording camera using SMART V2.5.21 program software (Panlab, Spain). A submerged transparent platform was placed $30 \mathrm{~cm}$ from the pool's edge and submerged $2 \mathrm{~cm}$ beneath the water surface. The water temperature was controlled at $22-24^{\circ} \mathrm{C}$. The platform remained in the same position throughout trials.

The platform was removed, and the rats were free to swim for 2 min to screen out the insensitive rats before the start of the experiment. As a result, only 75 healthy SD rats were selected for further experiments. The selected healthy rats were tested for the navigation experiment and the space exploration experiment.

Positioning navigation experiment: Rats were trained for 4 consecutive days to determine their learning ability. At the beginning of the training, starting points were randomly selected in the southeast and the northwest quadrants, and the rats were placed gently against the pool wall. A video system tracked and recorded the rats swimming in the maze. The time taken to find the platform was recorded as escape latency. If rats failed to reach the platform within $60 \mathrm{~s}$, the escape latency was recorded as $60 \mathrm{~s}$, and the rats were guided to the platform and left for $10 \mathrm{~s}$. Each rat was trained 4 times a day, 20-30 min for each interval, and the average latency across these trials was used as the latency value for each day. On the $5^{\text {th }}$ day, each animal was tested in four, single $60 \mathrm{~s}$ sessions from each quadrant.

Space exploration experiment: the main purpose was to measure the spatial memory ability of rats. After the 5th day of the water maze experiment, the platform was removed allowing the rats to swim freely for $60 \mathrm{~s}$. The duration spent in the target quadrant and the number of crossing times in the quadrant were recorded

\section{Material and Tissue Processing}

Blood samples $(4 \mathrm{ml})$ were collected from the inferior vena cava after abdominal anesthesia with $10 \%$ chloral hydrate $(0.3 \mathrm{ml} /$ $100 \mathrm{~g})$. All serum samples were separated, and stored in a $-70^{\circ} \mathrm{C}$ ultra low temperature freezer for later use.

All rats were then sacrificed to harvest hippocampus. Pre-chilled physiological saline was added to obtain 10\% hippocampal homogenate, and proteins extracted using RIPA lysates. $1 \mathrm{ml}$ RIPA lysate (containing 1mmol PMSF) was mixed with $100 \mu \mathrm{g}$ of sample and sonicated for $1 \mathrm{~min}$ (sonicated $2 \mathrm{~s}$ and interval $1 \mathrm{~s}$ ) in a $1.5 \mathrm{ml} \mathrm{EP}$ tube on the ice. All samples were then centrifuged at 2,500 $\mathrm{r} / \mathrm{min}$ for $10 \mathrm{~min}$ at $4^{\circ} \mathrm{C}$ to harvest supernatant, and stored in a $-70^{\circ} \mathrm{C}$ ultra low temperature freezer before use.

\section{Detection of BDNF Concentration by ELISA Method}

Levels of BDNF were determined using Thermo Scientific's Rat BDNF ELISA kit per the manufacturer's instructions. Briefly, the sample $(100 \mu \mathrm{l})$ was added to its appropriate well, and incubated 
for $2.5 \mathrm{~h}$ at room temperature. The solution was discarded, and the wells were washed 4 times with $1 \times$ Wash Buffer. Next, $100 \mu \mathrm{l}$ of $1 \times$ prepared biotinylated antibody was added to each well and incubated for $1 \mathrm{~h}$ at room temperature with gentle shaking. $100 \mu \mathrm{l}$ of prepared Streptavidin-HRP solution was then added to each well and incubated for $45 \mathrm{~min}$ at room temperature with gentle shaking. The solution was than discarded and the wells were washed 4 times with $1 \times$ Wash Buffer. Finally, $100 \mu$ l of TMB Substrate was added to each well and incubated for $30 \mathrm{~min}$ at room temperature in the dark with gentle shaking. Each plate was evaluated within $30 \mathrm{~min}$ of stopping the reaction. Optical density OD values were measured at the wavelength of $450 \mathrm{~nm}$, and the OD values of supernatant and serum in hippocampal homogenate were measured using a multi-function enzyme labelling analyzer.

\section{Ethics Statement}

All experiments were conducted in accordance with the animal experiment regulations of the Second Xiangya Hospital Ethics Committee of Central South University. The protocol was approved by the Second Xiangya Hospital Ethics Committee of Central South University.

\section{Statistical Analysis}

The statistical methods were analyzed by SPSS 22.0 software. One way ANOVA was used to analyze the data in accordance with normal distribution and homogeneity of population variance. Two way ANOVA and Bonferroni's multiple comparisons test were used to analyze the data of escape latency in water maze. LSD method was used for comparison between groups. The measurement data were expressed as mean \pm standard deviation $(\mathrm{X} \pm \mathrm{SD})$ or means \pm sem. For data not in accordance with normal distribution, Mann Whitney $\mathrm{U}$ method in rank sum test was used for inter group comparison, and the results were expressed by relevant parameters. P represents the significant level of the test, $\mathrm{P}<0.05$ means the difference is statistically significant.

\section{RESULTS}

\section{Quantitative Analysis of Experimental Animals}

All 75 rats (ketamine 3 d group $n=20$, saline 3 d group $n=18$, ketamine 14 d group $n=20$, saline 14 d group $n=17$ ) were included in the statistical analysis of the experimental results. There were no accidental deaths in the experiment. A total of 75 rats were screened by water maze.

\section{Experimental Results of Morris Water Maze}

The results from the Morris water maze are shown in Figure 1 and Table 1. The average escape latency of ketamine $3 \mathrm{~d}$ and $14 \mathrm{~d}$ groups were significantly longer than that of the saline $3 \mathrm{~d}$ and $14 \mathrm{~d}$ groups $(p<0.0001)$, respectively. The significant difference showed in day 3 comparing saline 3 days group with ketamine 3 days group by multiple comparison $(\mathrm{p}<0.05)$.

On day six, in the space exploration experiment, a significant reduction in the number of platform crossings as well as residence time was observed in the ketamine $3 \mathrm{~d}$ group compared to the saline $3 \mathrm{~d}$ group $(p<0.05)$. Compared to the saline $14 \mathrm{~d}$ group, the number of platform crossings and the

TABLE 1 | Statistical results of the number of crossing platforms and residence time in each group of rats in space exploration experiments $(X \pm S D)$.

\begin{tabular}{lccc}
\hline Group & Number & $\begin{array}{c}\text { The number of } \\
\text { crossing platform }\end{array}$ & $\begin{array}{c}\text { The residence time in the } \\
\text { target platform quadrant(s) }\end{array}$ \\
\hline ketamine 3 d & 20 & $6.5 \pm 2.0^{\mathrm{bd}}$ & $6.7 \pm 3.5^{\mathrm{bd}}$ \\
saline 3 d & 18 & $8.3 \pm 3.5^{\mathrm{a}}$ & $9.6 \pm 4.0^{\mathrm{a}}$ \\
ketamine14 d & 20 & $8.0 \pm 2.8^{\mathrm{d}}$ & $8.5 \pm 3.7^{\mathrm{d}}$ \\
saline 14 d & 17 & $10.0 \pm 2.7^{\mathrm{ac}}$ & $11.0 \pm 3.7^{\mathrm{ac}}$ \\
\hline
\end{tabular}

${ }^{a} P$ value comparison with the ketamine $3 d$ group, $P<0.05,{ }^{b} P$ value comparison with the saline $3 \mathrm{~d}$ group, $P<0.05,{ }^{c} P$ value comparison with the ketamine $14 \mathrm{~d}$ group, $P<0.05,{ }^{d} P$ value comparison with the saline $14 d$ group.
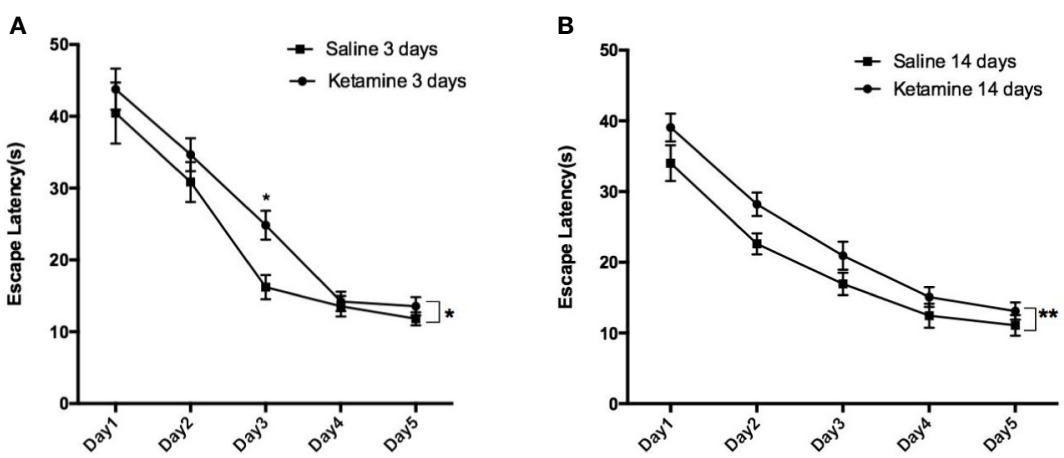

FIGURE 1 | The escape latencies of rats in water maze positioning navigation experiment. Data shown as means \pm sem. Rats were grouped by different treatment, Two-way ANOVA with Bonferroni correction were used to determine whether difference existed based on experience level. Significantly difference were seen between saline 3 days group vs. ketamine 3 days group as shown in (A), saline 14 days vs. ketamine 14 days group as shown in (B). Further analysis each group at different time point by Bonferroni's multiple comparisons test in Prism6, the significant difference showed in day3 comparing saline 3 days group with ketamine 3 days group. ${ }^{\star} \mathrm{p}<0.05,{ }^{\star *} \mathrm{p}<0.0001$. 


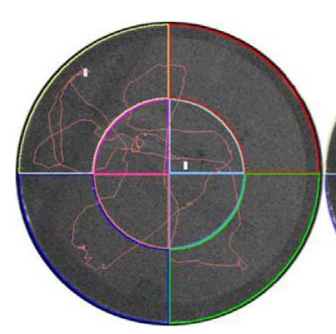

Ketamine 3 d group

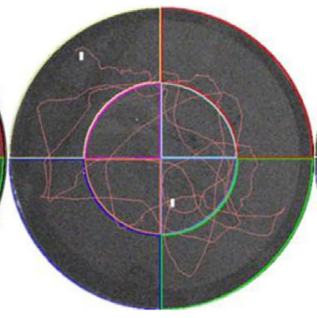

Ketamine 14 d group

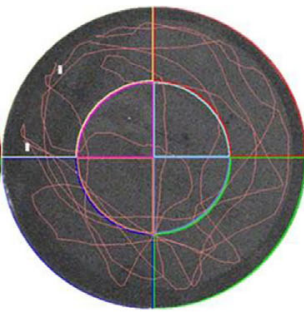

Saline 3 d group

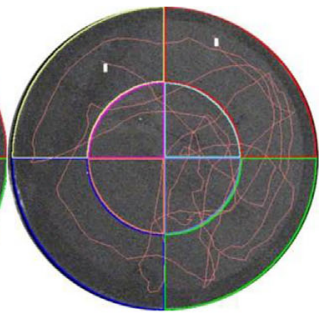

Saline 14 d group

FIGURE 2 | Typical trajectory map of four groups of rats in space exploration.

residence time was also significantly reduced in the ketamine $14 \mathrm{~d}$ group $(p<0.05)$. However, there was no significant differences observed between the ketamine $3 \mathrm{~d}$ and ketamine $14 \mathrm{~d}$ groups.

As showed in Figure 2, the track map of the target platform quadrant in the $3 \mathrm{~d}$ ketamine group is thinner than in the $3 \mathrm{~d}$ saline group. Similar results were also observed between the ketamine $14 \mathrm{~d}$ group and the saline group. Therefore, as observed in both the $3 \mathrm{~d}$ and $14 \mathrm{~d}$ group, there appears to be a memory deficit in the ketamine group when compared to saline controls.

\section{Results of BDNF Concentration in Serum and the Hippocampus}

The serum concentration of BDNF in the ketamine $14 \mathrm{~d}$ group was significantly lower than that of the saline $14 \mathrm{~d}$ group $(p<0.05)$. No significant difference was observed in serum BDNF concentration between the ketamine $3 \mathrm{~d}$ and saline $3 \mathrm{~d}$ group (Table 2).

BDNF concentrations in the hippocampus are showed in Table 3. No significant differences were found in hippocampal

TABLE 2 | Comparison of serum brain-derived neurotrophic factor (BDNF) concentration of each group.

\begin{tabular}{lccccc}
\hline Group & Total number & $\mathbf{U}$ & $\mathbf{W}$ & $\mathbf{Z}$ & $\mathbf{P}$ \\
\hline A vs B & 38 & 171.000 & 342.000 & -0.263 & 0.792 \\
C vs D & 37 & 88.000 & 299.000 & -2.469 & $0.014^{*}$ \\
A vs C & 40 & 183.000 & 393.000 & -0.460 & 0.646 \\
\hline
\end{tabular}

Group A represents ketamine 3 d group, Group B represents saline 3 d group, Group C represents ketamine14 d group, Group $D$ represents saline 14 d group, *indicates ketamine $14 d$ group compared with saline14 d group, $P<0.05$. $U, W$, and $Z$ value represent statistics. $P$ represents significant difference.

TABLE 3 | Comparison of hippocampus brain-derived neurotrophic factor (BDNF) concentration of each group.

\begin{tabular}{cccccc}
\hline Group & Total Number & $\mathbf{U}$ & $\mathbf{W}$ & $\mathbf{Z}$ & $\mathbf{P}$ \\
\hline A vs B & 38 & 175.000 & 385.000 & -0.146 & 0.884 \\
C vs D & 37 & 124.000 & 334.000 & -1.402 & 0.161 \\
A vs C & 40 & 199.000 & 409.000 & -0.027 & 0.987 \\
\hline
\end{tabular}

Group A represents ketamine 3 d group, Group B represents saline 3 d group, Group C represents ketamine14 d group, Group D represents saline 14 d group. U, W, and $Z$ value represent statistics. $P$ represents significant difference.
BDNF concentrations between any of the ketamine and saline groups (ketamine $3 \mathrm{~d}$ vs. saline $3 \mathrm{~d}$ : ketamine $14 \mathrm{~d}$ vs. saline $14 \mathrm{~d}$ ).

\section{DISCUSSION}

This experiment was designed to explore the neural mechanism related to ketamine addiction. Studies have found that ketamine abuse can lead to increased apoptosis of neurons in the frontal lobe, temporal lobe, hippocampus, among other regions, affecting cognitive function (1). Given that ketamine addiction can produce behaviors similar to schizophrenia, it has been suggested that ketamine addiction can be used as an experimental model of schizophrenia. Therefore, we assessed for differences in hippocampal and serum BDNF levels simultaneously to further elucidate the potential relationship between BDNF and ketamineinduced learning-memory dysfunction.

We first determined the effect of the acute and chronic ketamine $(25 \mathrm{mg} / \mathrm{kg})$ exposure on learning ability and memory in rats. Our results showed that the continuous use of ketamine for $3 \mathrm{~d}$ or $14 \mathrm{~d}$ both lead to learning and memory dysfunction. The concentration of BDNF in serum and hippocampal homogenate was also measured by ELISA. Interestingly, we only observed a significant difference in serum BDNF concentrations between the ketamine $14 \mathrm{~d}$ group and the saline $14 \mathrm{~d}$ group. Based on this, we further assessed the effect of ketamine on learning-memory dysfunction in rats and its relationship with BDNF.

A recent study showed that the effect of ketamine on cognition was directly related to the dosage $(6,15,16)$. However, there is no specific unity in the classification of low dose, subanesthetic dose and anesthetic dose among the present experimental literature. Low dose $(2.5-10 \mathrm{mg} / \mathrm{kg})$ ketamine can cause cognitive impairment, while lower doses $(<2.5 \mathrm{mg} / \mathrm{kg})$ and a slightly higher dose (10-20 $\mathrm{mg} / \mathrm{kg}$ ) do not significantly affect cognition after 10 consecutive days of exposure (16). Some researchers have even used higher doses of ketamine $(25 \mathrm{mg} / \mathrm{kg})$ to study the effects on rats and found that they lead to positive and negative symptoms similar to schizophrenia as well as affect cognitive function (17). However, Imre et al. (9), suggest that $4-16 \mathrm{mg} / \mathrm{kg}$ of ketamine is a subanesthetic dose for rodents, and $20-50 \mathrm{mg} / \mathrm{kg}$ is a high dose (18). Meanwhile, Newcomer et al. have shown that for rats, the minimum response threshold is $40 \mathrm{mg} / \mathrm{kg}$ (19). Previous studies 
have investigated the effect of ketamine on memory consolidation, and found that subanesthetic doses $(5-50 \mathrm{mg} / \mathrm{kg})$ did not affect the reconsolidation phase, whereas higher, anesthetic doses (125-150 $\mathrm{mg} / \mathrm{kg}$ ) did affect memory reconsolidation (15). Given the above findings, we chose the $25 \mathrm{mg} / \mathrm{kg}$ dose to study the effects of ketamine on learning and memory in rats. Consistent with previous studies, our results show that in the water maze experiment, the average latency of the ketamine- $14 \mathrm{~d}$ group was longer than that of the saline-14 d group. The number of platform crossings and the residence time of the rats in the $3 \mathrm{~d}$ and the $14 \mathrm{~d}$ ketamine groups were lower than that of the control groups. These results suggest that repeated exposure to $25 \mathrm{mg} / \mathrm{kg}$ ketamine negatively affects learning and memory in SD rats.

It has also been reported that the effect of ketamine on cognition is related to the duration of exposure as well as the time since last exposure. Single ketamine exposure and repeated ketamine exposure both cause cognitive impairments $(10,20)$. For example, when ketamine $(30 \mathrm{mg} / \mathrm{kg}$ ) was injected continuously for one month, the spatial memory ability of rats was significantly reduced (21).

In animal studies, a single exposure as well as 3-5 $\mathrm{d}$ of exposure have all been defined as acute ketamine exposure (8, $10,16,22)$. Likewise, the definition of chronic ketamine exposure varies between experimental studies. In fact, some have defined chronic ketamine exposure in rodents as $5 \mathrm{~d}$ (2), $7 \mathrm{~d}$ (17), $10 \mathrm{~d}$ (16), $14 \mathrm{~d}$ (23), and $30 \mathrm{~d}$ (21). Therefore, based on the literature, we defined $3 \mathrm{~d}$ ketamine exposure as "acute" and $14 \mathrm{~d}$ as a "chronic" in order to study the difference in cognition between acute and chronic ketamine exposure in rats.

The half-life of ketamine is about 1 to $2 \mathrm{~h}$, and the withdrawal reaction usually takes place between $0.5 \mathrm{~d}$ to $3 \mathrm{~d}$ (2). Most previous studies conducted the behavioral test $24 \mathrm{~h}$ after the last drug injection, thus not accounting for the effects of the withdrawal response. In order to avoid the effect of acute withdrawal in this experiment, the rats were given ketamine for $10 \mathrm{~d}$ after the last exposure. This study showed that acute and chronic ketamine $(25 \mathrm{mg} / \mathrm{kg})$ exposure both lead to learning and memory dysfunction in rats.

It had been reported that no matter acute or chronic exposure to ketamine, the damage to learning and memory of rats is persistent and irreversible $(16,23)$. In this experiment, the learning and memory abilities of rats were measured 10 days after ketamine withdrawal. Both the acute or chronic ketamine exposure led to significant impairment in cognitive dysfunction, comparing to control group.

$\mathrm{n}$ human and primates in general, cognition is mainly controlled by the prefrontal cortex and hippocampus, while rodents mainly depend on the function of the hippocampus (24). Importantly, deficiencies in BDNF is associated with hippocampal neuronal apoptosis. To verify whether learning-memory dysfunction in rats caused by ketamine is associated with BDNF deficiency in the hippocampus, we determined the levels of BDNF in the hippocampus of ketamine-treated rats. Interestingly, we found that there was no significant change in hippocampal BDNF concentrations after ketamine withdrawal. Intriguingly, we found that levels of BDNF were reduced in serum after chronic ketamine withdrawal. This suggests that serum BDNF levels are a better indicator of learning-memory dysfunction produced by ketamine exposure than hippocampal BDNF levels.
In conclusion, our study demonstrated that chronic ketamine (25 mg/kg) administration causes spatial learning and memory impairment in SD rats, and that this deficit might be associated with decreased serum BDNF levels. Additionally, our results also suggest that there are other mechanisms regulating BDNF levels in the brain. Nevertheless, it is still unclear how deficiencies in serum BDNF levels lead to learning and memory deficits in rats, requiring further exploration.

The limitation of this study is that we did not study the effects of ketamine addiction on human cognitive function and serum BDNF concentration. In the future, cognitive impairment caused by different doses and time of ketamine addiction patients can be measured with cognitive scale, and serum BDNF concentration can be measured at the same time. In order to understand the changes of brain structure, functional imaging of brain can be completed at the same time to understand the changes of neurotransmitters in the brain.

\section{DATA AVAILABILITY STATEMENT}

The raw data supporting the conclusions of this article will be made available by the authors, without undue reservation.

\section{ETHICS STATEMENT}

The animal study was reviewed and approved by the Second Xiangya Hospital Ethics Committee of Central South University.

\section{AUTHOR CONTRIBUTIONS}

HS, XZ, and ZX conceptualized the experiments. ML, YL, and QZ performed the experiments. $\mathrm{XZ}, \mathrm{SH}$, and $\mathrm{QH}$ undertook the statistical analysis. ML and AX wrote the first draft of the manuscript. XZ and YC reviewed and edited the manuscript. XZ, TS, XC, and ZL contributed to the final manuscript. All authors contributed to the article and approved the submitted version.

\section{FUNDING}

This work was supported by the National Natural Science Foundation of China (81971249), the National Basic Research Program of China (2015CB553504), the National Research Program of China (2016YFC0800908-Z02), and the National Natural Science Foundation of China (81501108).

\section{ACKNOWLEDGEMENTS}

Thanks my teachers and my classmates in the lab for the selfless help. 


\section{REFERENCES}

1. Taffe MA, Davis SA, Gutierrez T, Gold LH. Ketamine impairs multiple cognitive domains in rhesus monkeys. Drug Alcohol Depend (2002) 68:17587. doi: 10.1016/S0376-8716(02)00194-1

2. Ke X, Ding Y, Xu K, He H, Zhang M, Wang D, et al. Serum brain-derived neurotrophic factor and nerve growth factor decreased in chronic ketamine abusers. Drug Alcohol Depend (2014) 142:290-4. doi: 10.1016/j.drugalcdep.2014.06.043

3. Liang HJ, Lau CG, Tang A, Chan F, Ungvari GS, Tang WK. Cognitive impairments in poly-drug ketamine users. Addict Behav (2013) 38:2661-6. doi: 10.1016/j.addbeh.2013.06.017

4. Paule MG, Li M, Allen RR, Liu F, Zou X, Hotchkiss C, et al. Ketamine anesthesia during the first week of life can cause long-lasting cognitive deficits in rhesus monkeys. Neurotoxicol Teratol (2011) 33:220-30. doi: 10.1016/ j.ntt.2011.01.001

5. Farber NB, Wozniak DF, Price MT, Hayashi H, Dikkes PS. Age-specific neurotoxicity in the rat associated with NMDA receptor blockade: potential relevance to schizophrenia? Biol Psychiatry (1995) 38(12):788-96. doi: 10.1016/0006-3223(95)00046-1

6. Valentim AM, Ribeiro PO, Olsson IA, Antunes LM. The memory stages of a spatial Y-maze task are not affected by a low dose of ketamine/midazolam. Eur J Pharmacol (2013) 712:39-47. doi: 10.1016/j.ejphar.2013.04.027

7. Morgan CJ, Mofeez A, Brandner B, Bromley L, Curran HV. Acute effects of ketamine on memory systems and psychotic symptoms in healthy volunteers. Neuropsychopharmacology (2004) 29:208-18. doi: 10.1038/sj.npp.1300342

8. McNally JM, McCarley RW, Brown RE. Chronic Ketamine Reduces the Peak Frequency of Gamma Oscillations in Mouse Prefrontal Cortex Ex vivo. Front Psychiatry (2013) 4:106. doi: 10.3389/fpsyt.2013.00106

9. Tso MM, Blatchford KL, Callado LF, Mclaughlin DP, Stamford JA. Stereoselective effects of ketamine on dopamine, serotonin and noradrenaline release and uptake in rat brain slices. Neurochem Int (2004) 44(1):1-7. doi: 10.1016/S0197-0186(03) 00104-9

10. Li J, Wang B, Wu H, Yu Y, Xue G, Hou Y. 17beta-estradiol attenuates ketamineinduced neuroapoptosis and persistent cognitive deficits in the developing brain. Brain Res (2014) 1593:30-9. doi: 10.1016/j.brainres.2014.09.013

11. Iwasaki Y, Ishikawa M, Okada N, Koizumi S. Induction of a distinct morphology and signal transduction in TrkB/PC12 cells by nerve growth factor and brain-derived neurotrophic factor. J Neurochem (1997) 68:927-34. doi: 10.1046/j.1471-4159.1997.68030927.x

12. Ricci V, Martinotti G, Gelfo F, Tonioni F, Caltagirone C, Bria P, et al. Chronic ketamine use increases serum levels of brain-derived neurotrophic factor. Psychopharmacol (Berl) (2011) 215:143-8. doi: 10.1007/s00213-010-2121-3

13. Kauer JA, Malenka RC. Synaptic plasticity and addiction. Nat Rev Neurosci (2007) 8(11):844-58. doi: 10.1038/nrn2234

14. Ren Z, Sun WL, Jiao H. Dopamine D1 and N-methyl-D-aspartate receptors and extracellular signal-regulated kinase mediate neuronal morphological changes induced by repeated cocaine administration. Neuroscience (2010) 168(1):48-60. doi: 10.1016/j.neuroscience.2010.03.034
15. Wang JH, Fu Y, Wilson FA, Ma YY. Ketamine affects memory consolidation: differential effects in T-maze and passive avoidance paradigms in mice. Neuroscience (2006) 140:993-1002. doi: 10.1016/j.neuroscience.2006.02.062

16. Nikiforuk A, Popik P. The effects of acute and repeated administration of ketamine on attentional performance in the five-choice serial reaction time task in rats. Eur Neuropsychopharmacol (2014) 24:1381-93. doi: 10.1016/j.euroneuro.2014.04.007

17. Fraga DB, Reus GZ, Abelaira HM, De Luca RD, Canever L, Pfaffenseller B, et al. Ketamine alters behavior and decreases BDNF levels in the rat brain as a function of time after drug administration. Braz J Psychiatry (2013) 35:262-6. doi: 10.1590/1516-4446-2012-0858

18. Imre G, Fokkema DS, Den Boer JA, Ter Horst GJ. Dose-response characteristics of ketamine effect on locomotion, cognitive function and central neuronal activity. Brain Res Bull (2006) 69:338-45. doi: 10.1016/ j.brainresbull.2006.01.010

19. Newcomer JW, Farber NB, Jevtovic-Todorovic V, Selke G, Melson AK, Hershey $\mathrm{T}$, et al. Ketamine-induced NMDA receptor hypofunction as a model of memory impairment and psychosis. Neuropsychopharmacology (1999) 20:106-18. doi: 10.1016/S0893-133X(98)00067-0

20. Cao YL, Zhang W, Ai YQ, Zhang WX, Li Y. Effect of propofol and ketamine anesthesia on cognitive function and immune function in young rats. Asian Pac J Trop Med (2014) 7:407-11. doi: 10.1016/S1995-7645(14)60066-3

21. Huang S, Dai Y, Zhang Z, Hao W, Chen H. Docosahexaenoic acid intake ameliorates ketamine-induced impairment of spatial cognition and learning ability in ICR mice. Neurosci Lett (2014) 580:125-9. doi: 10.1016/j.neulet. 2014.08.011

22. Gastambide F, Mitchell SN, Robbins TW, Tricklebank MD, Gilmour G. Temporally distinct cognitive effects following acute administration of ketamine and phencyclidine in the rat. Eur Neuropsychopharmacol (2013) 23:1414-22. doi: 10.1016/j.euroneuro.2013.03.002

23. Nagy LR, Featherstone RE, Hahn CG, Siegel SJ. Delayed emergence of behavioral and electrophysiological effects following juvenile ketamine exposure in mice. Transl Psychiatry (2015) 5:e635. doi: 10.1038/tp.2015.111

24. Nakako T, Murai T, Ikejiri M, Ishiyama T, Taiji M, Ikeda K. Effects of a dopamine D1 agonist on ketamine-induced spatial working memory dysfunction in common marmosets. Behav Brain Res (2013) 249:109-15. doi: 10.1016/j.bbr.2013.04.012

Conflict of Interest: The authors declare that the research was conducted in the absence of any commercial or financial relationships that could be construed as a potential conflict of interest.

Copyright (c) 2020 Li, Xie, Liu, Zeng, Huang, Huang, Shao, Chen, Liao, Cai, Xiao, Zhang and Shen. This is an open-access article distributed under the terms of the Creative Commons Attribution License (CC BY). The use, distribution or reproduction in other forums is permitted, provided the original author(s) and the copyright owner(s) are credited and that the original publication in this journal is cited, in accordance with accepted academic practice. No use, distribution or reproduction is permitted which does not comply with these terms. 